\title{
Time Series Analysis in the Task of Oil Equipment Malfunctions Forecasting
}

\author{
Iakov S. Korovin, Maxim V. Khisamutdinov \\ Scientific research institute of multiprocessor computer systems at the Southern Federal University, Taganrog, Russia \\ korovin_yakov@mail.ru, L-V-P@yandex.ru
}

\begin{abstract}
The task of oilfield equipment malfunctions' early prevention is considered in the paper. Input data for the analysis are the indications of sensors, established in the well and represented as time series set. Due to the forecasting analysis (predictive analytics) of the given set it is possible to discover a new data vector, which will allow to classify the possible equipment failure and to eliminate it proactively. The main stage of this task is the prediction of a single time series. In this paper, we propose a new method of forecasting using $\mathrm{r} / \varphi$ - algorithm and the artificial neural network training algorithm (GAhNN) [1], previously developed by the authors. In the paper a new forecasting method, based on the $r / \varphi$ algorithm and the algorithm of artificial neural network training (GAhNN) [1], developed by authors before, is offered. Comparison with the known mostly applied forecasting algorithms - Kalman filter and ARIMA is fulfilled.

Index Terms - Forecasting, neural network, oilfield equipment, malfunction, Kalman filter.
\end{abstract}

\section{Introduction}

Oil and gas industry is crucially vital for Russian economy, that circumstance causes the constant appeal for new approaches, software and hardware, intended to increase the oil extraction process efficiency. One of most obvious ways to raise it - is to solve the task of preventing the oilfield equipment's failures [2]-[4]. Nowadays the amount of the information, concerning oil equipment work mode management can reach enormous values (approximately one million of parameters). In this regard, we propose to forecast the oilfield equipment state on the basis of the time series data analysis, whereas the input data are the oil well sensors indications.

\section{Research Methods and Approaches}

We denote the set of series as $S,|S|=n$, where $\mathrm{n}$ is the total number of series, describing oilfield equipment sensor data, $S_{i}$ - a series of data, describing the $i$-the sensor data, $i \in[1, n]$. In this paper the problem of oil production equipment mode forecasting on basis of the analysis of $S$ set is solved. The basic idea of the suggested approach is shown on the Fig . 1. On the first stage the sensor data acquisition is fulfilled which is made on the $k$ immersion depth and the $S$ set is formed. Next is the prediction of all elements of $S$ in order to obtain a list of predictive parameters for further analysis. Fig. 2 shows a fragment of the time series, describing the voltage data.

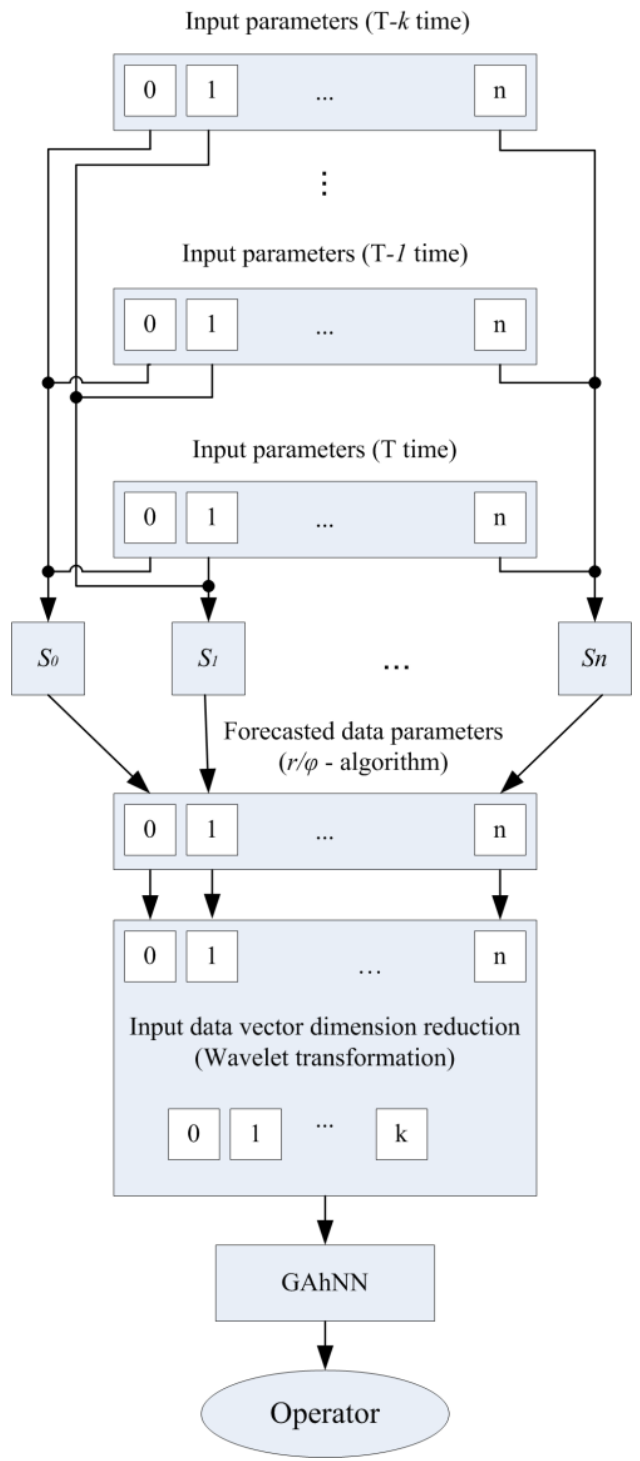

Fig. 1 Forecasting of oilfield equipment malfunctions' method.

\section{Time Series Forecasting Using R/ $\Phi$ Algorithm}

To predict the time series it is proposed to use a nonsmooth functions analysis based on $r / \varphi$ - the algorithm, proposed for summing divergent continued fractions [5], who found a variety of applications in computational mathematics [6]-[9]. 


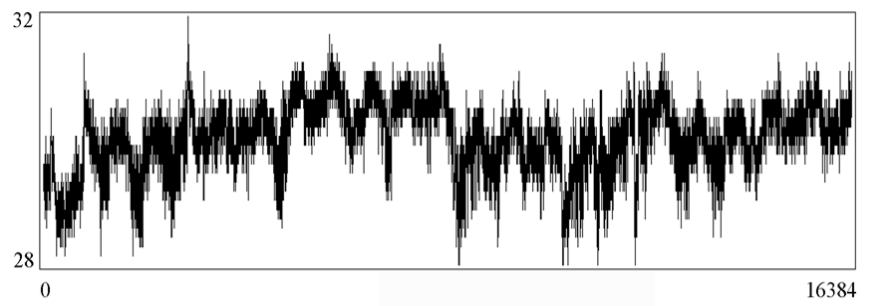

Fig. 2 Fragment of the time series describing the pumps' voltage.

Let us consider the above mentioned approach in brief. For the $S_{i}$ time series it is possible to get some characteristics which will be named $r / \varphi$ characteristics. For the $S_{i}$ time series let's introduce $r / \varphi$ characteristics.

$r$ - characteristics:

$$
r\left(S_{i}, k_{0}, k\right)=\sqrt[k]{\prod_{j=1}^{k}\left|S_{i, k_{0}+j}\right|},
$$

where $k_{0}$ is the beginning point for the $S_{i}$ time series segment vector, $k$ - is the length of the segment for which $r$ characteristic is calculated. Fig. 3 shows the $r$ characteristic diagram for the data fragment from the Fig. 2 $(\Delta=8192, n=[1 . .100+8192,8093 . .8192+8192])$ :

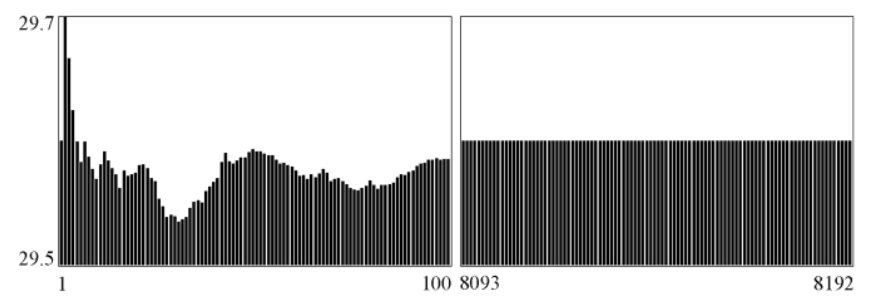

Fig. $3 r$ characteristics diagram.

$\Phi$ characteristics module:

$$
\left|\varphi\left(S_{i}, k_{0}, k\right)\right|=\pi \lim _{n \rightarrow \infty} \frac{k_{n}}{n},
$$

where $k_{n}$ - number of $S_{i}$ time series values less than $S_{i}$ beginning point from the total $k$ values, belonging to the $\left[S_{i, k 0}, S_{i, k 0+k}\right]$ segment. Fig. 4 represents the $\varphi$ characteristics diagram for the data fragment from Fig. 2. $(\Delta=8192, n=[1 . .100+8192,8093 . .8192+8192])$ :

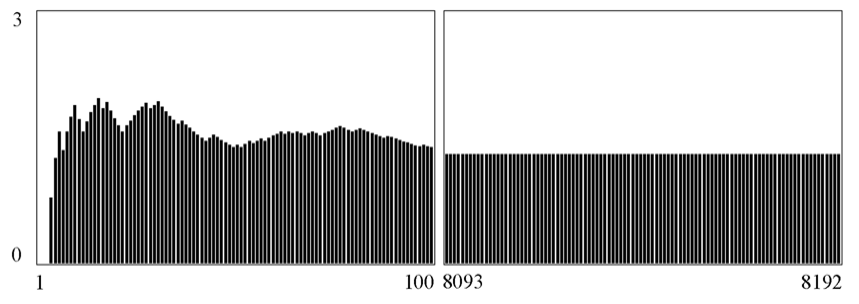

Fig. $4 \varphi$ characteristics diagram.
The next stage is $\Delta$ selection, initial point is fixed and the segment's expansion is proceeded (of which one that corresponds to delta). In the case where the conditions $\left|r \_n-r \_o\right|<e r r_{-} r$ and $\left|\varphi \_n-\varphi \_o\right|<e r r \_\varphi$ are true delta is fixed and the and the transition to the time series analysis window algorithm is taken over. The essence of the approach is calculation of $r / \varphi$ characteristics for each new value. In the case if there is reduction or increase of $r$ value or there's $\varphi$ exceeding $e r r_{-} r$ or $e r r_{-} \varphi$ we can announce that some event has occurred. On the overall analysis basis the training of the artificial network is undertaken, applying the approach, considered in [1]. Further on, taking into account calculated rand $\varphi$ values the forecasting of next $m_{-}$dat row element's values is made. Thus, the suggested forecasting algorithm scheme is as follows (Fig. 5).

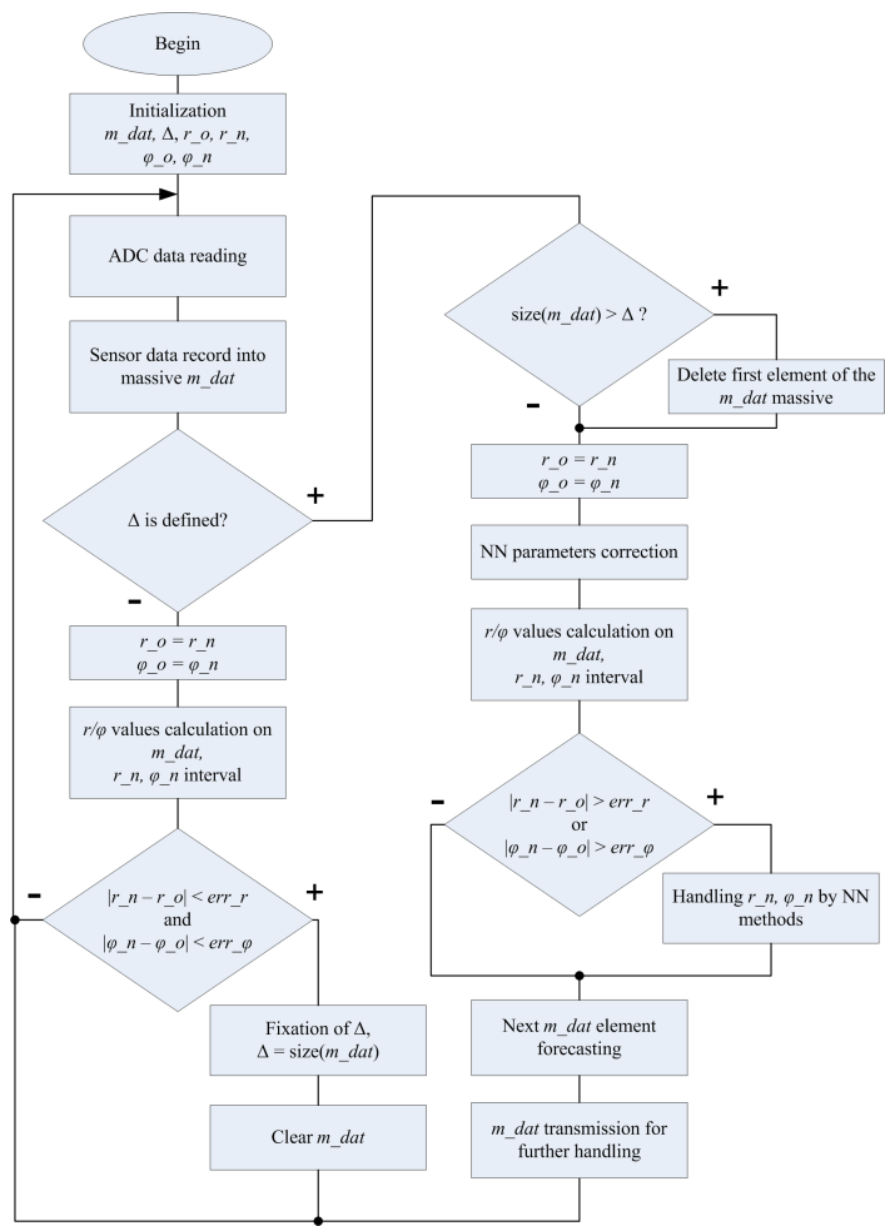

Fig. 5 The scheme of time series forecasting with use of $r / \varphi$ algorithm and GAhNN.

Further on the forecasting parameters vector $V_{\text {in }}$ is being handled with the application of the preliminary data processing algorithm, based on the wavelet analysis use with the aim of the input data vector dimension reduction. The efficiency of this approach is confirmed by the research, depicted in [1]. After that the received vector $V_{\text {out }}$ is transmitted to the classification block input. As there was shown in the authors' 
research [10], one of the most effective classification method is the GAhNN, proposed in [1]. The method is based on two Data Mining approaches - the artificial neural networks technology and the evolutionary approach. Taking this into consideration in the final stage of the oil equipment state's forecasting method the GAhNN approach is being applied.

\section{Experimental Research}

During the current research the authors have undertaken the experimental research of the proposed forecasting method with the use of $r / \varphi$ and GAhNN algorithms. The usnetelec time series benchmarks and the automated forecasting system [12], [13] were used for testing. Table I represents the comparison results of the proposed method with the existing ones, namely the Kalman recursive filter and ARIMA [12]. The results interference format is equal to the considered in [10].

TABLE I Comparison Results of the Proposed Method with Kalman Recursive Filter and ARIMA

\begin{tabular}{|c|c|c|c|c|}
\hline $\begin{array}{c}\text { Test } \\
\text { Samples }\end{array}$ & $\begin{array}{c}\text { Kalman } \\
\text { recursive filter }\end{array}$ & $\begin{array}{c}\text { ARIMA } \\
(0,1,1)\end{array}$ & $\begin{array}{c}\text { ARIMA }(2,1,2) \\
\text { with drift }\end{array}$ & $\begin{array}{c}r / \varphi \text { - algorithm } \\
\text { + GAhNN }\end{array}$ \\
\hline 2004 & 3898 & 3909 & 3969 & 3958 \\
\hline 2005 & 3956 & 3969 & 3970 & 3962 \\
\hline 2006 & 4017 & 4029 & 4097 & 4084 \\
\hline 2007 & 4073 & 4087 & 4112 & 4123 \\
\hline 2008 & 4121 & 4144 & 4219 & 4199 \\
\hline 2009 & 4193 & 4200 & 4254 & 4232 \\
\hline 2010 & 4225 & 4254 & 4343 & 4345 \\
\hline 2011 & 4269 & 4308 & 4393 & 4388 \\
\hline 2012 & 4311 & 4360 & 4470 & 4482 \\
\hline 2013 & 4357 & 4410 & 4529 & 4510 \\
\hline
\end{tabular}

As it can be seen from the Table I, in the majority of the samples ARIMA has represented the best results; the work results of the proposed forecasting method on the basis of the $r / \varphi$ algorithm and GAhNN combination are close to the ARIMA's ones and in some test samples exceed them. Thus the considered research confirms the suggested approach's application efficiency in the task of time series forecasting.

\section{Conclusions}

A method of the oil equipment's state forecasting with the times series analysis application is considered in the paper. This method is based on the authors' previous scientific results, in particular the artificial neural network training algorithm with the evolutionary approach usage (GAhNN) and the preliminary data handling applying wavelet transformations for the input vector data compression. As it was revealed during the research the before suggested GAhNN approach applied together with the $r / \varphi$ algorithm can de successfully used in the time series forecasting task. In comparison with the known forecasting algorithms it provides highly competitive results, and in some test samples exceeding ones. Further work can be intended to solve the task of the suggested forecasting algorithms' work speed increase applying parallel computing and distributed calculations.

\section{Acknowledgment}

The paper is published due the financial support of Russian Fund of fundamental research, projects 13-08-01172, 14-08-00776 and 14-08-00800.

\section{References}

[1] I.S. Korovin, M.V. Khisamutdinov, A.I. Kalyaev. The Application of Evolutionary Algorithms in the Artificial Neural Network Training Process for the Oilfield Equipment Malfunctions' Forecasting. 2nd International Symposium on Computer, Communication, Control and Automation (3CA 2013), Allerton Press, p. 253-257.

[2] I.S. Korovin, M.V. Khisamutdinov, M.G. Tkachenko. Forecasting of oilfield equipment work conditions with the application of evolutionary algorithms and artificial neural networks. Oil industry, Moscow, №12, 2013, p. 128-132.

[3] I.S. Korovin. Methods and tools of oilfield equipment's state diagnostics on the basis of modern Data mining technologies. Oil industry, Moscow, №1, 2007, p. 80-85.

[4] I.S. Korovin, G.A. Galuev, S.Y. Korovin. Intellectual decision adoption support systems for technological processes management in oil/gas production industry on the basis of neuronetwork and mutliagent technologies usage. Optical Memory and Neural Networks, Allerton Press, New York, USA, Issue № 3, 2006, p.179-185.

[5] V.I. Shmoylov. Periodical chain fractions, - Lvov: Academic express, 1998. -219 p.

[6] V.I. Shmoylov. Chain fractions. In 3 Volumes. Volume 2.National academy of sciences, Ukraine. Institute of applied problems of mechanics and mathematics. - Lvov: Mercator, 2004. - 558 p.

[7] V.I. Shmoylov. Chain fractions and $r / \varphi$ algorithm. -Taganrog: SFEDU, 2012. $-608 \mathrm{p}$

[8] I.I. Levin, M.V. Khisamutdinov, V.I. Shmoylov. The Weierstrass function and $r / \varphi$ - features // Gerald of Southern RAS scientific center., - 2014. - № 1 (150). - pp. 144-158.

[9] V.I. Shmoylov. Summation of chain fractions// Gerald of VSU.: Physics, Math. - 2013. - № 2. - pp 258-276.

[10] Rob J. Hyndman, Yeasmin Khandakar Automatic Time Series Forecasting: The forecast Package for R Vol. 27, Issue 3, Jul 2008

[11] Kalman R.E. (1960). A new approach to linear filtering and prediction problems. Journal of Basic Engineering 82 (1): pp. 35-45

[12] Asteriou, Dimitros; Hall, Stephen G. (2011). ARIMA Models and the Box-Jenkins Methodology. Applied Econometrics (Second ed.). Palgrave MacMillan. pp. 265-286. ISBN 978-0-230-27182-1.

[13] Iakov S. Korovin et al., 2014, Advanced Materials Research, Volume, 902, p.409, doi 10.4028/www.scientific.net/AMR.902.409, ISSN: 16628985 . 